\title{
The Polarization of the Political System in Albania and the Problems it has Caused
}

\section{Adelina Nexhipi}

Ph. D. Candidate, University of Tirana, History and Philology Faculty Email: adelinanexhipi@yahoo.com

\section{Doi:10.5901/mjss.2013.v4n9p151}

Abstract:

The Albanian transition, apart from the others, was passing from a party-state to a party pluralism. In Albania there are 123 political parties. Although the Albanian political life is dominate by the two biggest parties, the Socialist Party and the Democratic Party. The political debate among these two parties is characterized by high tones arrogance and from the total lack of democratic culture. There has been no readiness between them to do compromises, no desire to cooperate and no will to put the national interests over the partial ones. In this qualifying study I am going to describe an analytical panorama of essential political polarity in Albania, during the transition years based on the documents of the international institutions; Commission European, Council of Europe, OSCE-ODIHR and publications of foreign and Albanian authors. What are the peak moments of the political polarization in Albania? What kind of problems has this feature created for Albania? These are some of the question I am going to answer in this paper. From the made research it is arrived in the conclusion that the political polarity in Albania has created dislike and distrust in the people, prevented the progress of the country, the work of parliament and the European integrity of Albania.

Key words: political polarization, transition, cooperation, compromises,

\section{Introduzione}

L'Albania ha sofferto per quasi mezzo secolo di regime dittatoriale crudele, che si basava su un estremo accentramento politico ed economico. II Partito del Lavoro, e il suo leader, Enver Hoxha, avevano il monopolio del potere politico in Albania. Questo è stato approvato dalla Costituzione del 1976, che ha vietato l'attività di altri partiti politici. Secondo questa Costituzione l'ideologia marxista-leninista era l'unica ideologia dominante e la lotta di classe, la forza motrice della società (questo significherebbe guerra aperta contro ogni "nemico" del comunismo).

Nel 1990, le proteste popolari e le riforme realizzate dallo Stato hanno messo l'Albania sulla via della democratizzazione. Nella scena politica, sono apparse organizzazioni, movimenti e partiti politici con idee e programmi vari, il Partito Democratico, Socialdemocratico, Ecologico, Repubblicano, ecc. II 31 marzo 1991 in Albania si sono tenute le prime elezioni multipartitiche, le uniche dopo la Seconda Guerra Mondiale. La transizione verso la democrazia è stata molteplice; transizione politica - dal partito statale al pluralismo partitico; pluralismo economico - da un'economia centralizzata ad un'economia di mercato; transizione sociale - da un sistema chiuso e controllato in una società libera; transizione della sicurezza nazionale - da un Paese isolato ad un Paese che lavora per l'integrazione europea.

\section{Descrizione della situazione politica in Albania}

A partire dal 1990 fino ad oggi, in Albania, sono stati creati 123 partiti politici albanesi. Tuttavia la scena politica albanese è stata teatro di aspri e prolungati conflitti fra i due maggiori partiti del paese, il Partito Democratico e il Partito Socialista. Questi due sono passati l'un l'altro al potere dal 1992 fino ad oggi. La seguente tabella indica il numero di voti ottenuti dai due partiti nelle elezioni parlamentari, 1992-2009.

Tabella 1. Voti ottenuti da PD e PS nelle elezioni parlamentari in Albania (CEC)

\begin{tabular}{|c|c|c|c|c|}
\hline \multirow{2}{*}{$\begin{array}{c}\text { Data elezioni } \\
\text { parlamentari }\end{array}$} & \multicolumn{2}{|c|}{ Partito Democratico } & \multicolumn{2}{c|}{ Partito Socialista } \\
\cline { 2 - 5 } & $\%$ dei voti & $\begin{array}{c}\text { Seggi in } \\
\text { parlamento }\end{array}$ & \% dei voti & $\begin{array}{c}\text { Seggi in } \\
\text { parlamento }\end{array}$ \\
\hline 22 Marzo 1992 & 62.08 & 92 & 25.70 & 38 \\
\hline 26 Maggio 1996 & 55.50 & 122 & 20.40 & 10 \\
\hline
\end{tabular}




\begin{tabular}{|c|c|c|c|c|}
\hline 29 Giugno 1997 & 25.82 & 24 & 52.71 & 101 \\
\hline Giugno 2001 & & 46 & & 73 \\
\hline Giugno 2005 & 7.7 & 56 & 8.9 & 42 \\
\hline 28 Giugno 2009 & 40.18 & 68 & 40.85 & 65 \\
\hline
\end{tabular}

Secondo Freedom House del 2002, "le differenze tra le due parti, piuttosto che differenze programmatiche e ideologiche, sono legate ai cambiamenti nella personalità dei loro leader."

(http://www.freedomhouse.org/report/freedom-world/2002/albania, 2002)

Questi due partiti hanno maturato una lotta politica molto aspra, che molte volte supera i limiti di una cultura democratica. Al dibattito politico manca la comprensione, la tolleranza, il consenso tra le parti. In una democrazia il dibattito è essenziale, perché proprio esso rafforza ed espande la democrazia. E' abbastanza normale che il governo e l'opposizione abbiano opinioni e atteggiamenti diversi, molte volte contrari, poichè il dibattito stimola la crescita. Ma questo dibattito deve essere costruttivo e all'interno di norme etiche.

Il dibattito politico in Albania non si sviluppa come ideologia, o concetti politici, ma si concentra molto sul confronto tra la gente e a volte sembra prendere la forma di avversioni personali. Di questo punto di vista è anche Prof. Gjergj Sinani, il cui ha detto:" Per il fatto che i partiti non sono abbastanza consolidati a trasmettere agli elettori messaggi programmatici chiari, allora ci troviamo di fronte ad una caratteristica molto interessante, il dibattito politico tende verso atteggiamenti personali carismatici. Ironia della sorte, i vecchi modi di ragionare ereditati dal precedente regime, cioè la separazione della politica polarizzante "noi" ( i sostenitori della "linea giusta") e "loro" ("i nemici del popolo e del partito"), continua a sopravvivere. (Sinani, 2007)

La situazione politica in Albania rimane una preoccupazione e la polarizzazione partitica è alta. Retorica provocatoria e la mancanza di dialogo e consenso continuano a tenere alta la tensione politica. Questo panorama si nota di piu' durante il periodo delle campagne elettorali, periodo in cui la tensione è più alta e più duro il dibattito. I discorsi dei candidati durante le campagne sono pieni di accuse senza fine per la parte avversa, al posto di rivelare i loro programmi politici. Nel suo libro, l'Albania in transizione, Elez Biberaj descrive così la tensione durante i periodi elettorali: "Questi due partiti hanno visto le elezioni come un concorso dove il vincitore vince tutto e chi perde perde tutto, spesso ignorando le regole democratiche, manipolando le procedure elettorali, cercando di reprimere il sistema giudiziario e $i$ media, opponendo ogni esito sfavorevole per loro. " (Biberaj, 2011, p. 521)

La polarizzazione partitica e la mancanza di consenso tra i partiti politici in Albania è stato notato più volte dalle istituzioni internazionali, considerando la mancanza di tolleranza e di comprensione come i roditori che corrodono la democrazia.

- La relazione della Commissione di Venezia sulla visita del suo presidente, Antonio La Pergola in Albania, 8-10 gennaio 1998 ha dichiarato: "Impossibile il dialogo politico. Questo rende difficile trovare una soluzione alla situazione in cui si trova il paese.E' necessario prendere decisioni appropriate per le elezioni politiche e legislative allo scopo di portare il Paese fuori dalle difficoltà economiche, ansia sociale e incertezza del diritto". (http://www.venice.coe.int/docs/1998/CDL(1998)053-e.asp, 1998)

- Nel rapporto del 2005 della Commissione Europea per l'Albania, tra le altre cose si dice: "Il clima politico in Parlamento ha continuato ad essere problematico. Diverse sessioni plenarie si sono svolte in un clima molto conflittuale, dove i due campi opposti non sono stati in grado di mostrare uno spirito costruttivo ... "

- La relazione del 2007 della Commissione Europea, ha osservato: "le dispute politiche hanno ritardato le elezioni amministrative del 2007 e hanno portato a molte carenze tecniche."

- La relazione della Commissione Europea per l'Albania, del 2010 evidenzia anche la mancanza di dialogo politico in Albania e la necessità di trovare un compromesso tra i partiti politici del paese. Nel rapporto c'è scritto: "La forte cultura della politica di parte polarizza le opinioni in parlamento, questo ostacola il buon funzionamento dell'Assemblea e delle sue commissioni ... Non c'è consenso tra i partiti in Parlamento riguarda il funzionamento del parlamento e gli standard da seguire.... L'attività parlamentare, sia in termini di emanare leggi, cosi come esercitazione delle funzioni di vigilanza, per molti anni è stato caratterizzato da un dialogo profondamente difficile e spesso non costruttivo. II lavoro del Parlamento è stato oscurato e minato dallo stallo politico." (http://www.mie.gov.al)

In questi 20 anni di pluralismo politico in Albania, le forze politiche continuano in interotamente le loro lotte e si sono rifiutati di collaborare e di risolvere i grandi problemi del paese. Di seguito elenchiamo alcuni dei momenti in cui la polarizzazione partitica e la mancanza di un consenso hanno realmente causato gravi problemi per il paese:

- Approvazione della Costituzione, la legge fondamentale di uno Stato. Nonostante la sua necessità per 
l'approvazione, i partiti politici non hanno raggiunto un accordo tra di loro. La mancanza di consenso tra le parti ha fatto che l'Albania è stato l'ultimo paese dell'ex blocco comunista, il quale ha approvato una costituzione propria. Ma ancora una volta la sua approvazione non è stata raggiunta con il consenso di tutte le parti.

- Il suo picco, la polarizzazione dei partiti, è arrivato alla fine del 1996 - inizio del 1997 e in combinazione con il malcontento popolare hanno provocato una grave crisi economica, sociale e politica nel 1997. Elez Biberaj descrive la situazione politica in Albania nel corso del 1997: "Mentre la retorica oscilava tra dure dichiarazioni di arroganza ed espressioni di volontà di compromesso, i Socialisti e i Democratici non hanno mostrato alcuna volontà di assumersi la responsabilità comune per risolvere i problemi del paese ". (Biberaj, 2011, p. 470) Prof. Valentina Duka, riferendosi a questo periodo, scrive: "I rapporti tra le forze politiche continuavano ad essere tesi. Dopo gli eventi nel 1997, il PS e il PD mostravano un'ostilità ancora più profonda verso l'un l'altra. Di conseguenza, non si è mai concretizzato nessun tipo di cooperazione, cosi' fondamentale ed utile." (Duka, 2007, p. 362)

- Il dibattito politico feroce e l'impossibilità di trovare un terreno comune fra le parti, hanno portato nelle proteste anti-governative innescate dall'opposizione nel 1998, che degenerarono in scontri tra manifestanti e forze di polizia.

- All'inizio del 2004 l'opposizione organizzò delle proteste anti-governative (proteste contro il governo Nano, con lo slogan "Nano vattene"), accusando il governo di brogli elettorali e di non aver adottato le riforme necessarie per migliorare gli standard di vita.

- Il divario tra le forze politiche in Albania è apparso nelle elezioni parlamentari parziali del 2007. Costanti dibattiti partitici hanno portato ad un boicottaggio da parte dell'opposizione delle elezioni legislative parziali, nella primavera di quest'anno.

- La tensione nella vita politica, l'aumento della sfiducia tra le parti, l'aggravamento del dibattito politico, hanno spinto l'opposizione ad organizzare proteste popolari anti-governative, il 21 gennaio 2011, in cui sono stati uccisi quattro manifestanti.

- Le elezione dei due ultimi presidenti, Bamir Topi e Bujar Nishani, sono state realizzate in una situazione di tensione. I partiti politici non hanno raggiunto il consenso di un unico candidato, il quale avesse il sostegno di tutti i partiti. Anche se in conformità con la Costituzione, le elezioni presidenziali non sono riuscite a soddisfare le aspettative sull' inclusione, così come non ha aiutato nel consolidamento del dialogo e della cooperazione politica. Questo ha portato ad un temporaneo rallentamento della riforma nei settori chiavi, laddove necessitava il consenso politico.

- Poiché non si riesce a trovare un linguaggio comune in termini di riforma elettorale, non esiste ancora una soluzione incontrastata riguardo a questo problema.

Così, ripetutamente, la politica albanese non è sfuggita alla situazione di stallo in cui entrava, grazie alle dispute e controversie che coinvolgono le principali forze politiche del paese. Nei momenti difficili di dibattito politico, la tentazione di usare il boicottaggio è stata continua. II boicottaggio è stato utilizzato da entrambe le parti, come una forma di non conformità con la situazione politica nel paese, in contrasto con le azioni o le decisioni della controparte, come rifiuto di sostenere le iniziative della parte avversaria,ecc.

- Nel corso dei dibattiti sulla Costituzione albanese (discussione durata diversi anni), il Partito Socialista ha boicottato il parlamento. II 17 giugno 1993 ha interroto i lavori in parlamento dichiarando che tornerebbe nel caso il progetto di Costituzione fosse pronto. Intanto durante questo processo il Partito Democratico ha boicottato i lavori della commissione parlamentare per la stesura della Costituzione, nel 1998. Secondo il PD il parlamento esistente (nato dalle elezioni politiche anticipate del 1997) non aveva l'autorità morale di approvare una Costituzione permanente. II Partito Democratico ha anche boicottato il referendum popolare per l'approvazione della Costituzione, il 22 novembre 1998, e ha invitato tutti i suoi sostenitori di non parteciparvi.

- II Partito Democratico ha boicottato il parlamento nel 1998, poiché il governo ha deciso di perseguire i sei ex funzionari del governo per uso il gas mortale allo scopo di reprimere le proteste del 1997.

- $\quad$ II Partito Democratico ha boicottato il secondo turno delle elezioni amministrative del 2000.

- Dopo le elezioni parlamentari del giugno 2001 fino al gennaio 2002, I'opposizione destra ha boicottato il parlamento a causa di irregolarità elettorali, secondo lei.

- II Partito Democratico alla fine del 2003 ha boicottato il parlamento per protestare contro l'atteggiamento del governo e l'esito delle elezioni locali dell' ottobre 2003 
- Le elezioni legislative parziali del 2007 sono state boicottate dall'opposizione (Partito Socialista) a causa dei dibattiti in continuità.

- L'opposizione socialista ha boicottato il parlamento dopo le elezioni del giugno 2009 per contestare il risultato di queste elezioni e ignorare questi esiti. L'opposizione ha concluso il boicottaggio parlamentare nel giugno 2010.

- In seguito delle elezioni amministrative del 2011 e le varie problematiche che sono state create in relazione al conteggio dei voti per la città di Tirana, il Partito Socialista ha boicottato il parlamento. Questo boicottaggio continuò fino al settembre dello stesso anno.

\section{I problemi causati al Paese dall'estrema polarizzazione politica}

II mancato raggiungimento di un accordo sulle regole del gioco politico ha alimentato l'ostilità e la violenza, portando via ogni speranza per il consolidamento della democrazia in Albania.

I partiti hanno allontanato le persone con i conflitti interni e i conflitti inutili tra di loro. Questo parere sostiene Mr. Björn von Sydow, Presidente per gli affari politici del Consiglio d'Europa, nell' intervista pubblicato al giornale "Panorama", il 7 ottobre 2011, dove si esprime: "La scena politica albanese dominata dalla polarizzazione partitica ha frustrato e deluso gli albanesi negli ultimi decenni. La situazione politica appare congelata nel suo aspetto da quasi 20 anni. I due principali partiti politici non hanno mostrato la volontà politica di raggiungere un consenso su questioni fondamentali." (Partite te kalojne ne veprime konkrete, 2011)

Questo fatto si nota anche nei dati preliminari del sondaggio di SOROS, condotto in Albania nel periodo dicembre 2012 - febbraio 2013. La tabella dei dati ed il grafico seguente mostrano com'è la fiducia nelle istituzioni albanesi e gli enti locali. Dai risultati preliminari del sondaggio viene capito che gli albanesi hanno poca fiducia in queste istituzioni e la fiducia nei politici e i partiti è inferiore a quella di altre istituzioni e le parti analizzate.

Tabella 2. Fiducia nelle istituzioni / attori locali (SOROS, 2013, p. 4)

\begin{tabular}{|c|c|c|}
\hline \multicolumn{3}{|c|}{$(0=$ affato $/ 10=$ piena fiducia $)$} \\
\hline Fiducia nella polizia & Le aree urbane & Le zone rurali \\
\hline La fiducia nel sistema legale & 4.5 & 5.4 \\
\hline Fiducia in parlamento & 3.2 & 3.9 \\
\hline Fiducia nei partiti politici & 2.8 & 3.1 \\
\hline Fiducia nei politici & 2.2 & 2.4 \\
\hline
\end{tabular}

Figura 1. Fiducia nelle istituzioni / attori locali

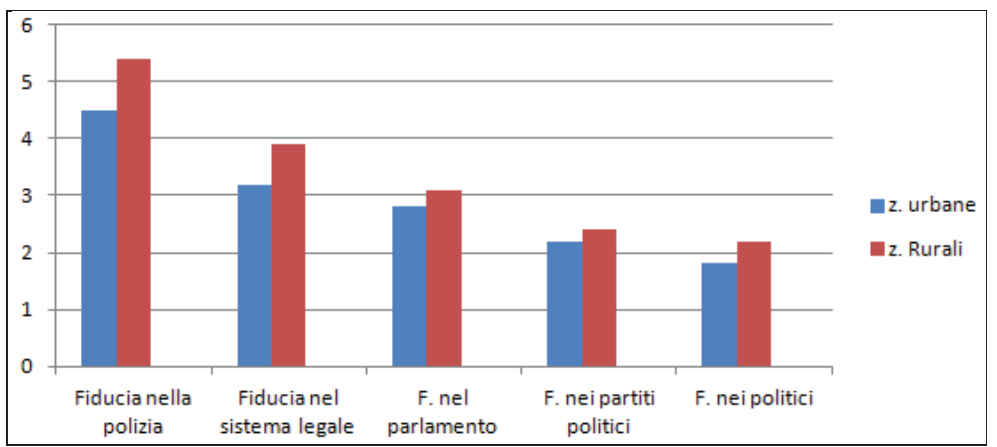

Ma, secondo i risultati preliminari di questo questionario, alla domanda -" Con quale partito ti senti più vicino?" |l 47,8\% degli intervistati ha risposto che non si sentono vicini a nessun partito, solo il 19,6\% di loro si sentono vicini al Partito Socialista e il 22,7\% si sente più vicino al Partito Democratico. (SOROS, 2013, p. 14) La diminuzione è anche verso interesse per la politica, in generale, il 34\% degli intervistati non sono interessati alla politica. Nella classifica europea, fatta da Soros, l'Albania è classificata al quinto posto, mentre al primo posto è il Portogallo, dove il $42 \%$ degli intervistati 
D'altra parte, questa costante polarizzazione ha ostacolato il processo di integrazione europea dell'Albania. La polarizzazione politica molto evidente in Albania è spesso un ostacolo al progresso del paese e potrebbe compromettere gli sforzi verso l'adesione all'Unione Europea - dice l'analista di problemi balcanici, Janusz Bugajski, in un'intervista con la Voice of America. Qui egli afferma: - C'è il rischio che la polarizzazione porti in una tale scissione del partito, che nulla possa essere approvata in Parlamento, che le principali questioni, come ad esempio la lotta contro la criminalità e la corruzione non siano riguardati, che la legislazione necessaria per completare questa lotta non si applichi, che il dibattito pubblico sia cosi politicizzato, da non permette lo svolgimento del lavoro legislativo. Questo è un problema per l'Albania, cosi come per gli altri paesi, e può ostacolare il progresso verso l'adesione all' UE. (http://www.pressonline.com.mk/ default-al)

Questo problema lo mette in rilievo anche il rapporto della Commissione europea,del 2007, che ha dichiarato: "C'è stato un progresso limitato per poter arrivare in un consenso costruttivo tra i partiti politici ad attuare le riforme necessarie per l'esecuzione del processo di stabilizzazione e di associazione" (http://www.mie.gov.al)

Albania ancora non ha ancora trovato la sua strada, in termini di una proficua collaborazione tra le forze politiche, di prendere decisioni e di avere un programma comune per il bene del paese e verso l'integrazione euro-atlantica. All'Albania è stato negato per tre volte consecutive la richiesta per lo status di paese candidato e una delle ragioni principali per questo è lo stallo politico che sorge nel paese e impedisce la realizzazione delle riforme. Quindi, la classe politica non ha dimostrato di avere la capacità di raggiungere una cooperazione politica, libero da interferenze esterne, mettendoli su un interesse individuale o di parte, gli interessi della nazione.

L'estrema polarizzazione politica, l'inadeguato livello di disponibilità a fare compromessi e i boicottaggi frequenti (analizzato in precedenza) ha impedito l'organo legislativo di svolgere il suo lavoro in modo corretto. I periodi di stallo politico nella primavera-estate del 2006, fermarono i tentativi del Parlamento di affrontare adeguatamente le questioni importanti delle riforme, soprattutto quelle che richiedono un ampio consenso politico. II clima politico debole ha ostacolato il lavoro dei gruppi parlamentari sulla riforma elettorale e gli accordi politici lenti hanno ritardato la nomina dei membri della Commissione Elettorale Centrale. Ciò ha portato ritardi per attuare le raccomandazioni dell'OSCE/ODIHR in seguito alle elezioni parlamentari del 2005. Questa fatto viene costantemente ripetuto in ogni rapporto della Commissione Europea sull' Albania:

- Nella relazione del 2005, ha dichiarato: "Il clima politico in Parlamento ha continuato ad essere problematico. Diverse sessioni plenarie si sono svolte in un clima molto conflittuale, dove i due campi opposti non sono stati in grado di mostrare una predisposizione costruttiva riguarda le leggi importanti. Nonostante l'intensificazione dell'attività parlamentare, l'atmosfera, in alcuni casi, ha determinato un rallentamento o prevenuto iniziative legislative fondamentali".

- Questo si ripete nel rapporto del 2007: "Il lavoro parlamentare spesso è dominato da un programma politico a breve termine, che ha polarizzato il dibattito. Le decisioni parlamentari sono spesso in ritardo a causa di dispute incentrate su interessi stretti partitici, di preparazione affrettata, o discussioni inadeguate. Questo ha rallentato le riforme, in particolare la riforma elettorale e della giustizia. "

- "L'attività parlamentare, per quanto riguarda l'approvazine delle leggi, e l'esercitazione delle funzioni di supervisione, da molti anni è profondamente caratterizzato da un dialogo difficile e spesso distruttivo." -viene detto nel corso del rapporto, nel 2010. (http://www.mie.gov.al)

A causa del dibattito politico molto aspro e la mancanza di compromesso nel Parlamento albanese è stato spesso difficile approvare leggi che richiedono una maggioranza. Questo ha creato ostacoli per il lavoro del Parlamento in elaborazione delle leggi, e come una reazione a catena, si bloccava l'impresa di riforme importante, lo sviluppo del paese, la crescita e il benessere, ecc. Prof. Piro Misha, accusa la classe politica della povertà del paese e dei problemi sociali. Egli scrive: "Albania, il paese che essi [i leader politici albanesi] hanno già governato da anni come presidenti, primi ministri, viceministri, ministri, parlamentari, capi di partiti, continua ad essere non solo uno dei paesi più poveri e sottosviluppati, ma è malato e confuso, un luogo dove quasi tutti vogliono fuggire, come in fuga da una nave che viene affondata! Allora, di chi è la colpa? " (Misha, 2009, p. 173)

Come viene accennato in precedenza, la polarizzazione politica diventa più intensa e sentita nei periodi elettorali, che si svolgono in un clima di polarizzazione e diffidenza. Secondo i rapporti, I'OSCE/ODIHR in Albania non si sono mai realizzati, dal 1991 ad oggi, elezioni pienamente democratiche, secondo gli standard europei. Da un'elezione ad altra 
sono state fatte progressi, ma non abbastanza da definirle libere e democratiche. II rapporto dell'OSCE/ODIHR, sulle elzioni dell' 8 maggio 2011 scrive: "Anche se le elezioni sono state trasparenti e competitive, erano altamente polarizzate, con diffidenza tra i partiti politici al potere e l'opposizione. Come nelle precedenti elezioni, i due maggiori partiti non svolgono i loro compiti elettorali in modo responsabile, influenzando negativamente la gestione delle elezioni. Disaccordi taglienti su base del partito sono stati osservati anche nella Commissione Elettorale Centrale." (OSCE, http://www.osce.org/albania, 2011)

Questa stessa frase si trova in qualsiasi rapporto dell'OSCE/ODIHR sulle elezioni politiche in Albania. Dopo la certificazione dei risultati delle elezioni, il dialogo politico si fa più difficile. Il partito che non ha vinto le elezioni inizia un fiume di accuse verso la parte avversaria per aver manipolato le elezioni, commercializzare i voti, alterare i risultati, ecc, ecc. Dalle dichiarazioni di cui sopra, in materia di boicottaggi, si capisce che i risultati delle elezioni in Albania sono stati sempre contestati dal partito che va all'opposizione. Ė diventata pratica comune per i partiti politici in Albania. Gli unici casi in cui la rotazione politica è avvenuta in modo abbastanza tranquilla, sono quelli del 1992 e del 2005. Così, dopo 22 anni di pluralismo politico e di elezioni pluralistiche in Albania, il paese continua a soffrire di "incubo dalle elezioni".

La polarizzazione politica e il dibattito violento tra i partiti politici si riflettono anche nel lavoro della Commissione Elettorale Centrale (CEC), i cui membri sono eletti dal Parlamento. I membri di questo comitato che sono eletti come rappresentanti di partiti politici, riflettono nell'attività del CEC il battibecco politico tra i partiti. Questo è stato notato anche nelle relazioni dei processi di valutazione elettorale dell'OSCE / ODIHR. Abbiamo il rapporto di valutazione sulle elezioni parlamentari del 2009: "CEC spesso era diviso secondo le linee politiche di maggioranza-minoranza, in particolare sulle questioni più controverse... Questa politicizzazione ha avuto un impatto sul lavoro del CEC, in particolare al suo dovere di amministrare le elezioni in modo neutrale ed efficiente." (OSCE, 2009, p. 9) II seguente ha continuato: "Durante le lamentele post-elettorali, le divisioni politiche all'interno del CEC sono diventate ancora più evidenti che nel periodo preelettorale. I membri della CEC e i rappresentanti dei partiti hanno fatto dichiarazioni politiche e osservazioni inutilmente lunghi e personali, piuttosto che facendo riferimento ai fatti e ai questioni procedurali." (Ibid, p. 29)

Questo fatto non manca neanche nelle relazioni della Commissione Europea, "Il risultato delle elezioni per il sindaco di Tirana è stato molto controverso e le decisioni della Commissione Elettorale Centrale (CEC) sono stati presi in spirito di parte, mancava la razionalità e avevano discrepanze "- si dice nella relazione intermedia del 2011. (http://www.mie.gov.al)

Quindi, è chiaro che la polarizzazione politica albanese e gli scontri continui e feroci tra i principali partiti politici del paese hanno causato grossi problemi, hanno ostacolato lo sviluppo del paese ed hanno disorientato la società. La polarizzazione politica è espressa in varie forme, dai boicottaggi parlamentari ai conflitti violenti tra i gruppi politici. La polarizzazione politica ha perfino preso forme estreme. Tale è il caso dell'aggressione e del ferimento di Azem Hajdari, (deputato e vice presidente del Partito Democratico al momento) il 18 settembre 1997 nella sede dell'Assemblea nazionale dal deputato socialista, Gafur Mazreku. Il gesto è stato fatto per ragioni personali piuttosto banali, eppure era a causa della forte tensione politica tra i due partiti. La polarizzazione politica e la mancanza di volontà delle forze politiche di risolvere i loro problemi in modo pacifico ha continuato a provocare la perdita di vite innocenti, come il caso del 21 gennaio 2011, quando quattro manifestanti delle proteste antigovernative organizzate dall'opposizione, sono stati uccisi.

\section{Conclusioni e raccomandazioni}

- La polarizzazione estrema politica sta danneggiando il funzionamento della democrazia in Albania e sta impedendo importanti riforme che sono necessarie per il progresso del paese sulla strada verso l'integrazione nella CE e il funzionamento dello stato di diritto comunitario.

- La situazione politica rischia di provocare la polarizzazione profonda e prolungata della classe politica, con gravi conseguenze per la disponibilità al compromesso e trovare consenso sulle riforme chiavi.

- II dialogo costruttivo tra le parti è necessario per garantire il buon funzionamento del parlamento sulla base delle sue regole.

- II dialogo politico è sempre peggio e di più, spesso conflittuale e totalmente improduttivo o inesistente.

- La forte cultura della politica di parte polarizza i posizioni in Parlamento e questo complica il buon funzionamento dell'Assemblea e delle sue commissioni .Lo stallo politico e l'impedimento del lavoro in parlamento, approfondisce la debole vigilanza parlamentare.

I politici albanesi devono: 
- raggiungere un ampio consenso politico e mettere gli interessi nazionali sopra le agende stretti e apparteneze partitiche,

- trovare un approccio pragmatico, in cui le opportunità e le sfide del Paese sono discussi in modo equilibrato e neutrale. Ciò richiede una responsabilità piena di tutte le forze politiche del paese,

- lavorare in maniera seria e sistematica allo scopo di rafforzare la democrazia, di aumentare la fiducia nelle persone che l'Albania ha tutte le capacità di costruire uno standard di democrazia europea,

- imparare ad essere tolleranti e disposti al compromesso, rispettare i risultati positivi dei governi precedenti, per potarli oltre e rafforzare la democrazia.

In conclusione tramite questo studio spero di aver dato un contributo, anche se picolo, nell' analisi di questo fenomeno della vita politica in Albania lancio un invito ai sociologi e ai politologi di studiare l' influenza della tradizione comunista sugli approcci dei lider politici albanesi e l'influenza del loro comportamento sull'atteggiamento dell'elettoratto albanase.

\section{Bibliografia}

Biberaj, E. (2011). Shqiperia ne tranzicion. Tirane, Albania: AllS.

CEC. (n.d.). http://www.cec.org.al/endex.php?option=com content\&view=article\&id=247:rezultatet. Retrieved from http://www.cec.org.al Duka, V. (2007). Histori e Shqiperise 1912-2000. Tirane, Albania: Kristalina-KH.

http://www.freedomhouse.org/report/freedom-world/2002/albania. (2002). Retrieved from http://www.freedomhouse.org/.

http://www.mie.gov.al. (n.d.). Retrieved from http://www.mie.gov.al.

http://www. pressonline.com.mk/default-al. (n.d.). Retrieved from http://www.pressonline.com.

http://www.venice.coe.int/docs/1998/CDL(1998)053-e.asp. (1998). Retrieved from http://www.venice.coe.int.

Misha, P. (2009). Arratisja nga burgjet e historise. Tirane, Albania: Toena.

OSCE. (2009). http://www.osce.org/albania. Retrieved from http://www.osce.org/albania.

OSCE. (2011). http://www.osce.org/albania. Retrieved from http://www.osce.org/albania.

Partite te kalojne ne veprime konkrete. (2011, Ottobre 7). Panorama .

Sinani, G. (2007, Novembre). Shqiperia sot, analiza dhe mundesi. Polis .

SOROS. (2013, Marzo 26). http://www.soros.al. Retrieved from http://www.soros.al. 